\title{
The applications of computer simulation technology in tennis serve technique
}

\author{
Wu Guo-dong, Jiang Xing-jian, Lin Li-jun
}

Science and Technology College, Nanchang Hangkong University; Nanchang 330034 China

wgd_1234567@163.com

Keywords: Computer Simulation Technique; Tennis; the height of hit tennis; serve's effect

\begin{abstract}
This article made Computer Simulation Technique to analysis the relationship of the different heights of hit tennis and serve's effect in the course of tennis serve. The results show that with the increase height of hit tennis the effective area of tennis serve also increased significantly; the success rate of serve higher and the angle of serve bigger; the flight time of tennis more short and the aggressiveness of serve stronger; the coach and athlete that they had master grasped serve technique should be more emphasis increase the effect of serve by raising the height of hit tennis.
\end{abstract}

\section{Introduction}

Tennis serve is the only mastered by herself without controlled techniques by opponent and the strong arms of tennis players to defeat the opponent. It is the beginning of each point and attack other. If you want to achieve the advantages of serving, you have only the best technology is not enough; the scientific philosophy of serve is also needed. The process of serve is influence by many facts, it is difficult to obtain the relevant research data in practice, but computer simulation technology can help ours to overcome these difficulties. We can make a model of serve by computer simulation technology, then research the model and achieve the relevant data. The data can be referenced by reform of serve technique and serve philosophy.

\section{Research Objects and Methods}

Research Objects.3D Model of Tennis Serve

Research Methods .The Method of Literature: Reading on a lot of materials about computer simulation technology and tennis serve;The Method of Depth Interviews: By visiting some tennis coaches and sports, understand their view and experience;The Method of Experiment: First make of the 3D software to design a model of tennis serve, then endow to the correspond data; finally go to analysis the data of experiment.;The Method of Mathematical Statistics: Using CAD software to process the experimental data, such as: us the factions of length calculation, area calculation and angle calculation to obtain further experimental data; us EXECL software and SPSS software to statistics and analysis for finally the experimental data.

\section{Experimental Designs}

3D Mod. according the information of The International Tennis Federation to establish the model of tennis court; Determine the position of hit tennis, to the right area as example. As show in Fig. 1 the $O$ point; Set the initial height of hit tennis was $2.92 m$;Draw the line $\mathrm{L}$ as a reference in the point of distance the net $3.5 \mathrm{~cm}$ 


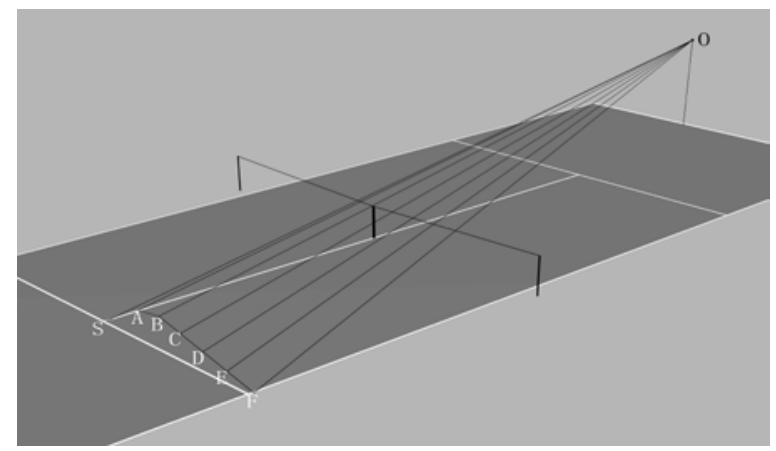

Figure 1 Graphic Model of 3D

\section{Experiment 1. The Influence of the Height of Hit Tennis to serve's Effect}

1) Set the height of $O$ was $2.92 \mathrm{~m}$.

2) From $O$ drawing a line to the serve middle-line, the point is assumed to $A$, the line is $O A$. Then adjustment $O A$ and make the point $A$ to slide in serve middle line. When the line $O A$ just across the line $\mathrm{L}$ the point $A$ is the limiting position from $O$ to the serve middle line.

3) Connect the $O$ and the lowest point of the line $L$ and extend, the point of contact with the tennis court is $B$. The point is the limiting position from $O$ just through the lowest point of the line $L$.

4) From $O$ drawing a line to the serve side-line, the point is assumed to $F$, the line is OF. Then adjustment $O F$ and make the point $F$ to slide in serve side-line. When the line $O F$ just across the line $L$ the point $\mathrm{F}$ is the limiting position from $O$ to the serve side-line.

5) From $O$ drawing three lines to the area of $B F$, then adjust the three lines to just through the line $\mathrm{L}$ and extend, the points of contact with the tennis court is $C, D$ and $E$.

6) Connect the points $A$ to $B, C, D, E$, and $F$. Then the effective service area is determined.

7) Us the $C A D$ software to draw the ichnography of the effective service area, calculate the area and record. As show in figure 2

8) Set the height of $O$ was $2.95 m, 2.98 m, 3.01 m, 3.04 m, 3.07 m, 3.10 m, 3.13 m, 3.16 m, 3.19 m$ and $3.22 \mathrm{~m}$, then repeat 1 ) to 8 ).

9) Adjust the point $F$ in the limiting position of exterior angle, then adjust the height of point $O$ and record the height of point $O$ when the line $O F$ just through the line $L$.

10) Adjust the point $A$ in the limiting position of interior angle, then adjust the height of point $O$ and record the height of point $O$ when the line $O A$ just through the line $\mathrm{L}$.

11) Adjust the point $B$ in the limiting position of the serve side-line, then adjust the height of point $O$ and record the height of point $O$ when the line $O A$ just through the lowest point of the line $L$.

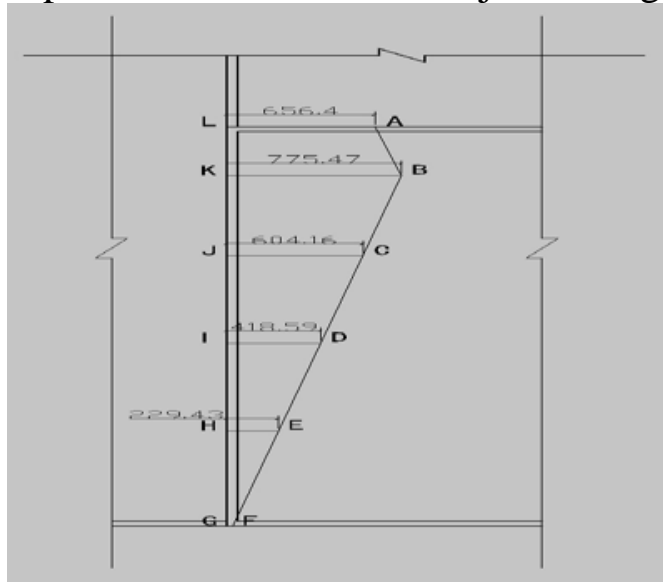

Figure 2 the Ichnography of the Effective Service Area

Experiment 2. The Influence of the Height of Hit Tennis to Flight Time of Tennis. Suppose the initial speeds of tennis are $50 \mathrm{~m} / \mathrm{s}$ at all height; Suppose every tennis hit to the same point of S; In all height draw the line OS from point $\mathrm{O}$ to point $\mathrm{S}$, then measure the angle $\angle$ a of OS and the vertical line of point $\mathrm{O}$ and record. 


\section{Results and Analysis}

The Influence of the Height of Hit Tennis to the effective service area. As show in table 1:1) the effective service area with the height of hit tennis increasing. 2) The increased range of effective service area is greater than the increased height of hit tennis. So athletes should be encouraged to improve the serving aggressiveness by raising the height of hit tennis.

Table 1. the relationship of the height of hit tennis and the effective service area

\begin{tabular}{|c|c|c|c|}
\hline$H \quad(m)$ & $S\left(m^{2}\right)$ & $\Delta S\left(m^{2}\right)$ & $\begin{array}{c}\text { The increased } \\
\text { rate of } S(\%)\end{array}$ \\
\hline 2.92 & 1.82 & & \\
\hline 2.95 & 2.18 & 0.36 & 19.60 \\
\hline 2.98 & 2.52 & 0.70 & 38.72 \\
\hline 3.01 & 2.89 & 1.07 & 58.71 \\
\hline 3.04 & 3.21 & 1.39 & 76.56 \\
\hline 3.07 & 3.54 & 1.72 & 94.63 \\
\hline 3.10 & 3.85 & 2.03 & 111.71 \\
\hline 3.13 & 4.16 & 2.34 & 128.62 \\
\hline 3.16 & 4.46 & 2.64 & 144.98 \\
\hline 3.19 & 4.76 & 2.94 & 161.45 \\
\hline 3.22 & 5.07 & 3.25 & 178.44 \\
\hline
\end{tabular}

Note: $H$ is the height of hit tennis, $S$ is the effective service area, $\triangle S$ is the area difference, the increased rate of $S$ is the ratio of $\triangle S$ and $S 1$.

The Influence of the Height of Hit Tennis to the point of serve. As show in table 2: 1) When the height of hit tennis is $2.91 \mathrm{~m}, \mathrm{FG}$ is zero. This shows that the tennis is difficult to be served to the side-line by athletes, when the height of hit tennis less than this height. 2) When the height of hit tennis is $2.71 \mathrm{~m}, A L$ is zero. This shows that the tennis is difficult to be served to the middle-line by athletes, when the height of hit tennis less than this height. 3) When the height of hit tennis is $2.68 \mathrm{~m}$, $B K, A L$ and $F G$ are all zero. This shows that the tennis can not be served to the effective service area by athletes, when the height of hit tennis less than this height.4) As $B K>A L>F G$, so the success rate of serve is the serve to body> exterior angle> interior angle.

Table 2. The Relation of the Height of Hit Tennis and the Effective Service Area

\begin{tabular}{|c|c|c|c|}
\hline$H(m)$ & $A L(\mathrm{~mm})$ & $B K(\mathrm{~mm})$ & $F G(\mathrm{~mm})$ \\
\hline 2.68 & 0 & 0 & 0 \\
\hline 2.71 & 0 & 132.93 & 0 \\
\hline 2.91 & 636.604 & 747.342 & 0 \\
\hline 2.92 & 656.396 & 775.467 & 27.518 \\
\hline 2.95 & 753.49 & 856.994 & 118.88 \\
\hline 2.98 & 838.615 & 942.58 & 209.053 \\
\hline 3.01 & 923.334 & 1022.713 & 300.721 \\
\hline 3.04 & 1002.396 & 1097.107 & 384.705 \\
\hline 3.07 & 1079.818 & 1172.787 & 469.697 \\
\hline 3.10 & 1154.486 & 1245.756 & 548.279 \\
\hline 3.13 & 1230.318 & 1313.299 & 631.025 \\
\hline 3.16 & 1299.369 & 1384.768 & 704.674 \\
\hline 3.19 & 1368.361 & 1453.396 & 782.744 \\
\hline 3.22 & 1437.732 & 1531.896 & 854.982 \\
\hline
\end{tabular}

\section{The Influence of the Height of Hit Tennis to Flight Time of Tennis}

Because tennis is impacted by the gravity and air resistance in the flight during, in general, air resistance is very small and the flight direction is constantly changing, so ignore. Therefore, according to the formula 
$V_{Y}=V \cos \angle a$

$H=V_{Y} t+1 / 2 g t^{2}$

The flight time of tennis is obtained.

As show in table 3, when the initial velocity and the point of tennis are all same, the flight time of tennis is decreasing; with the height of hit tennis is increasing. In this way, the response time of competitor is reduced in the race, and the aggressiveness of serve is stronger.

Table 3 the Relation of the Height of Hit Tennis and Flight Time of Tennis

\begin{tabular}{|c|c|c|}
\hline$H(m)$ & $\angle a^{\circ}$ & $T(S)$ \\
\hline 2.95 & 80.724 & 0.308242 \\
\hline 2.98 & 80.632 & 0.306031 \\
\hline 3.01 & 80.539 & 0.303847 \\
\hline 3.04 & 80.447 & 0.301689 \\
\hline 3.07 & 80.354 & 0.299558 \\
\hline 3.10 & 80.262 & 0.297584 \\
\hline 3.13 & 80.169 & 0.295503 \\
\hline 3.16 & 80.077 & 0.293448 \\
\hline 3.19 & 79.985 & 0.291417 \\
\hline 3.22 & 79.892 & 0.289411 \\
\hline
\end{tabular}

Note: $T$ is the flight time of tennis.

\section{Conclusion}

1) With the increase height of hit tennis the effective area of tennis serve also increased significantly.

2) The success rate of serve higher and the angle of serve bigger.

3) The flight time of tennis more short and the aggressiveness of serve stronger.

4) The coach and athlete that they had master grasped serve technique should be more emphasis increase the effect of serve by raising the height of hit tennis.

\section{References}

[1] Yan Botao. Biomechanica Analys is of the Serve Techniques in Tennis [J]. Sport Science, 2001(10):36 - 39

[2] Jiang Zhongwei. Research on Increasing the Flat Serve Rate in Tennis[J].Sport Science,2000, 20(4): $41-43$

[3] Polly1Davey ,Rodd1Thorpe and Clyde Williams1 Fatigue decrease skilled tennis performance [J ]Journal of sport science, 2002 （20）: $311-3181$

[4] Tao Zhixiang. Exploration on Tennis Service System[J ].Journal of Beijing Sport University,2004, 27 (12) :1695-1697

[5] Jin Chunlin. A Biomechanical Analysis of Bai Yan’s Tennis Service Technique[J]. Journal of Beijing Sport University, 2008, 31 (2) : 271-274 